\title{
Twenty-eight Pregnant Women with Cardiac Diseases: A Review of Maternal and Fetal Outcomes in Pakistan
}

\author{
Shoaibunnisa Soomro ${ }^{1}$, Raj Kumar ${ }^{2}$, Altaf A. Shaikh ${ }^{3}$ \\ 1. Obstetrics and Gynecology, Ghulam Muhammad Mahar Medical College and Hospital, Sukkur, PAK 2. Cardiology, \\ Ghulam Muhammad Mahar Medical College and Hospital, Sukkur, PAK 3. Internal Medicine, Ghulam Muhammad \\ Mahar Medical College and Hospital, Sukkur, PAK
}

Corresponding author: Shoaibunnisa Soomro,drshoaibunnisasoomro@gmail.com

\section{Abstract}

\section{Introduction}

In the western world, $0.2 \%-4 \%$ of pregnancies are complicated by heart diseases. The United Kingdom Obstetric Surveillance System (UKOSS) conducted a study on acute myocardial infarction (MI) in pregnant women. It estimated an incidence of 0.7 cases of acute MI per 100,000 pregnancies. In this study, we review the maternal and fetal outcomes of pregnant women with a cardiac disorder.

\section{Methods}

This retrospective analysis included all maternal records of the women registered in the obstetrics and gynecology department of Ghulam Muhammad Meher Medical Hospital, Sukkur, from January 1 to December 31, 2018. The study was approved by the institutional review board.

\section{Results}

The most common cardiac disease among our patients was peripartum cardiomyopathy ( $\mathrm{n}=12 ; 42.9 \%)$ followed by rheumatic heart disease in nine (32.1\%) women. The rate of maternal mortality was $14.2 \%(n=4)$. There were eight (28.6\%) cases of intrauterine devices (IUDs) and the remaining 20 (71.4\%) babies were born alive and healthy.

\section{Conclusion}

The prevalence of cardiac diseases in pregnancy in Pakistan is comparable to that in our neighboring countries. These cardiac diseases are responsible for fetal and maternal adverse outcomes.

Received 10/27/2019

Review began 10/28/2019 Review ended 10/28/2019 Published 10/30/2019

๑) Copyright 2019

Soomro et al. This is an open access article distributed under the terms of the Creative Commons Attribution License CC-BY 3.0., which permits unrestricted use, distribution, and reproduction in any medium, provided the original author and source are credited.
Categories: Cardiology, Obstetrics/Gynecology

Keywords: pregnancy, pregnancy complications, cardiomyopathy in pregnancy, pakistan, pakistan

\section{Introduction}

In the western world, $0.2 \%-4 \%$ of pregnancies are complicated by heart diseases [1]. The United Kingdom Obstetric Surveillance System (UKOSS) conducted a study on acute myocardial infarction (MI) in pregnant women. It estimated an incidence of 0.7 cases of acute MI per 100,000 pregnancies [2]. Acquired heart diseases are becoming more common in pregnancy due to older age at the time of first pregnancy and higher frequency of predisposing cardiovascular (CV) risk factors, including hypertension (HTN), diabetes mellitus (DM), and obesity [3]. Another important reason is that the advancements in medical care have allowed more women with cardiac diseases to conceive and carry pregnancy till term [4].

In developing countries, rheumatic heart diseases (RHD) constitute as many as $56 \%-89 \%$ CV co-morbidities during the antepartum period [5]. In an Indian study, 69\% of cases of CV co-morbidity during pregnancy were due to RHD [6]. In Pakistan, 1\% of pregnancies are complicated by cardiac diseases and as with India, $69 \%$ are caused by RHD [7].

Peripartum cardiomyopathy (PPCM) is the idiopathic failure of the myocardium. It occurs during the last month of pregnancy and may persist until five to six months of the postpartum period. Some women may develop signs of PPCM right after delivery or within a month postpartum rather than during the pregnancy [8]. The incidence of PPCM has been reported from many countries and has been highest in Nigeria (1/102 deliveries) and lowest in Japan (1/15,533 births) [9]. In Pakistan, younger women with higher parity and pregnancy-induced hypertension (HTN) are at high risk of PPCM [10].

Despite this, there are very few studies available in Pakistan focused especially on heart diseases in 
pregnancy, including clinical symptoms and radiological findings, in any part of the country. The aim of our study was to highlight the clinical and radiological findings and fetal and maternal outcomes in such patients so high-risk patients should be identified and more-focused treatment could be given to them.

\section{Materials And Methods}

This retrospective analysis included all maternal records $(n=2,282)$ of the women registered in the obstetrics and gynecology department of Ghulam Muhammad Meher Medical Hospital, Sukkur, from January 1 to December 31, 2018. The study was approved by the institutional review board.

Among all obstetric records, women with CV co-morbidity were identified and their patient records were extracted for review. Their type of CV co-morbidity, chest X-ray findings, electrocardiogram (ECG) and echocardiography (echo) findings, and maternal and fetal outcomes were included in this report. Data were presented as a tabulated form, and frequency and percentages were calculated.

\section{Results}

During the study period, 2,282 obstetric cases were booked for delivery in the department. CV co-morbidity was established in $28(1.2 \%)$ cases. Out of these, 21 (75\%) were diagnosed with CV co-morbidity during pregnancy and the remaining 7 (25\%) had chronic CV co-morbidity. The most common cardiac disease among our patients was peripartum cardiomyopathy $(n=12 ; 42.9 \%)$ followed by rheumatic heart disease in nine (32.1\%) women. Eighteen (64.4\%) women out of 28 were unbooked.

The rate of maternal mortality was $14.2 \%(n=4)$. All four women had PPCM. The baby of only one of these four women was born alive and the remaining three were intrauterine deaths (IUD). There were eight (28.6\%) cases of IUD and the remaining 20 (71.4\%) babies were born alive and healthy. Among these cases of IUD, four mothers had PPCM, two had RHD, one had pre-eclampsia, and one had ischemic heart disease (IHD).

A detailed report of the chest X-ray, ECG, and echo findings, along with the maternal and fetal outcomes of these cases are shown in Table 1.

\begin{tabular}{|c|c|c|c|c|c|c|}
\hline $\begin{array}{l}\text { S. } \\
\text { No }\end{array}$ & $\begin{array}{l}\text { Type of Cardiac } \\
\text { Disease }\end{array}$ & ECG Finding & X-ray Findings & Echo Findings & $\begin{array}{l}\text { Maternal } \\
\text { Outcome }\end{array}$ & $\begin{array}{l}\text { Fetal } \\
\text { Outcome }\end{array}$ \\
\hline 1 & $\begin{array}{l}\text { Peripartum } \\
\text { cardiomyopathy }\end{array}$ & $\begin{array}{l}\text { LA + LV } \\
\text { enlargement }\end{array}$ & $\begin{array}{l}\text { Increased CT ratio, increased pulmonary } \\
\text { congestion }\end{array}$ & $\begin{array}{l}\text { Dilated all } 4 \text { chambers, } \\
\text { global LV, hyperkinesias }\end{array}$ & Alive & Alive \\
\hline 2 & Pre-eclampsia & $\begin{array}{l}\mathrm{LVH}+\mathrm{LA} \\
\text { enlargement }\end{array}$ & $\begin{array}{l}\text { Increased CT ratio, increased pulmonary } \\
\text { congestion, upper tube diversion }\end{array}$ & $\begin{array}{l}\text { EF: } 35-40 \% \text {, dilated all } 4 \\
\text { chambers, moderate } \\
\text { generalized depressed LV } \\
\text { function }\end{array}$ & Alive & IUD \\
\hline 3 & $\begin{array}{l}\text { Rheumatic heart } \\
\text { disease }\end{array}$ & $\begin{array}{l}\text { RAD, RVH, LA/LV } \\
\text { enlargement }\end{array}$ & $\begin{array}{l}\text { Straightening of LHB with double contour } \\
\text { shadow on RHB, upper lobe diversion }\end{array}$ & $\begin{array}{l}\text { Severe MS, mild MR, EF: } \\
70 \%\end{array}$ & Alive & Alive \\
\hline 4 & Mitral stenosis & $\begin{array}{l}\text { RAD, RVH, LA } \\
\text { enlargement }\end{array}$ & $\begin{array}{l}\text { Straightening of LHB with double contour } \\
\text { shadow on RHB, upper lobe diversion }\end{array}$ & $\begin{array}{l}\text { Severe MS, calcified valves, } \\
\text { normal size LV, normal } \\
\text { function }\end{array}$ & Alive & Alive \\
\hline 5 & $\begin{array}{l}\text { Acute } \\
\text { pulmonary } \\
\text { edema }\end{array}$ & $\begin{array}{l}\text { P-mitrale, dilated } \\
\text { Ram RV, } \\
\text { depressed RV } \\
\text { function }\end{array}$ & $\begin{array}{l}\text { Straightening of LHB, double contour shadow } \\
\text { on RHB, upper lobe diversion, batwing } \\
\text { appearance, severe pulmonary congestion }\end{array}$ & $\begin{array}{l}\text { Severe mitral RAD stenosis, } \\
\text { dilated LA, EF: } 70 \%\end{array}$ & Alive & Alive \\
\hline 6 & $\begin{array}{l}\text { Rheumatic heart } \\
\text { disease }\end{array}$ & $\begin{array}{l}\text { P-mitrale, dilated } \\
\text { LV, severe LV } \\
\text { dysfunction }\end{array}$ & $\begin{array}{l}\text { Increased CT ratio with bilateral obliteration } \\
\text { of costophrenic angles, severe pulmonary } \\
\text { congestion }\end{array}$ & $\begin{array}{l}\text { EF: } 25 \% \text {, thickened mitral } \\
\text { valve, moderate MS and } \\
\text { severe MR }\end{array}$ & Alive & IUD \\
\hline 7 & $\begin{array}{l}\text { Peripartum } \\
\text { cardiomyopathy }\end{array}$ & $\begin{array}{l}\text { LA + LV } \\
\text { enlargement }\end{array}$ & $\begin{array}{l}\text { Increased CT ratio with pulmonary } \\
\text { congestion }\end{array}$ & $\begin{array}{l}\text { Dilated all } 4 \text { chambers with } \\
\text { severe generalized LV } \\
\text { dysfunction, EF: } 25-35 \%\end{array}$ & Alive & Alive \\
\hline 8 & $\begin{array}{l}\text { Peripartum } \\
\text { cardiomyopathy }\end{array}$ & $\begin{array}{l}\text { P-mitrale, LA + } \\
\text { LV enlargement }\end{array}$ & $\begin{array}{l}\text { Increased CT ratio with pulmonary } \\
\text { congestion, obliterated both costophrenic } \\
\text { angles, batwing appearance }\end{array}$ & $\begin{array}{l}\text { Dilated LA \& LV, severe LV } \\
\text { dysfunction }\end{array}$ & Alive & Alive \\
\hline 9 & $\begin{array}{l}\text { Peripartum } \\
\text { cardiomyopathy }\end{array}$ & $\mathrm{AF}$ & $\begin{array}{l}\text { Increased CT ratio with pulmonary } \\
\text { congestion, mild pleural effusion on right side }\end{array}$ & $\begin{array}{l}\text { Dilated LV, severe LV } \\
\text { dysfunction, Dilated LA }\end{array}$ & Alive & Alive \\
\hline
\end{tabular}




\section{Cureus}

\begin{tabular}{|c|c|c|c|c|c|c|}
\hline 10 & $\begin{array}{l}\text { Peripartum } \\
\text { cardiomyopathy }\end{array}$ & $\begin{array}{l}\text { LVH \& LA } \\
\text { enlargement + } \\
\text { LAD }\end{array}$ & $\begin{array}{l}\text { Dilated LA \& LV with increased } \\
\text { vasoconstriction, mild pleural effusion on } \\
\text { right side }\end{array}$ & $\begin{array}{l}\text { Dilated LV with global } \\
\text { hyperkinesias, EF:25-30\% }\end{array}$ & Alive & Alive \\
\hline 11 & $\begin{array}{l}\text { Peripartum } \\
\text { cardiomyopathy }\end{array}$ & AF & $\begin{array}{l}\text { Normal CT ratio with prominent aortic } \\
\text { knuckle }\end{array}$ & $\begin{array}{l}\text { Concentric hypertrophied LV } \\
\text { with normal EF }\end{array}$ & Alive & Alive \\
\hline 12 & $\begin{array}{l}\text { Peripartum } \\
\text { cardiomyopathy }\end{array}$ & $\begin{array}{l}\text { LA \& LV } \\
\text { enlargement }\end{array}$ & $\begin{array}{l}\text { Dilated LA \& LV, double contour shadow on } \\
\text { RHB, right-sided pleural effusion }\end{array}$ & $\begin{array}{l}\text { EF: } 20-25 \% \text {, Dilated LF with } \\
\text { generalized hypokinesia }\end{array}$ & $\begin{array}{l}\text { Maternal } \\
\text { death }\end{array}$ & IUD \\
\hline 13 & $\begin{array}{l}\text { Rheumatic heart } \\
\text { disease }\end{array}$ & $\begin{array}{l}\text { P-mitrale, LVH, } \\
\text { LAD }\end{array}$ & $\begin{array}{l}\text { Enlarged CT ratio with increased vascularity, } \\
\text { batwing appearance }\end{array}$ & $\begin{array}{l}\text { Generalized LV dysfunction, } \\
\text { EF: } 20-25 \%\end{array}$ & Alive & Alive \\
\hline 14 & $\begin{array}{l}\text { Ischemic heart } \\
\text { disease }\end{array}$ & $\begin{array}{l}\text { Poor } R \text { wave in } \\
\text { V1-V6, LAD }\end{array}$ & $\begin{array}{l}\text { Increased CT ratio with pulmonary } \\
\text { congestion }\end{array}$ & $\begin{array}{l}\text { Mildly dilated LV with apical } \\
\text { hypokinesia, EF: } 35-40 \%\end{array}$ & Alive & IUD \\
\hline 15 & $\begin{array}{l}\text { Peripartum } \\
\text { cardiomyopathy }\end{array}$ & $L V H+L A D+A F$ & $\begin{array}{l}\text { Increased CT ratio with increased vascularity, } \\
\text { dilated pulmonary arteries }\end{array}$ & $\begin{array}{l}\text { Dilated LV, gen. LV } \\
\text { dysfunction, EF: } 20-25 \%\end{array}$ & $\begin{array}{l}\text { Maternal } \\
\text { death }\end{array}$ & IUD \\
\hline 16 & $\begin{array}{l}\text { Cardiogenic } \\
\text { shock }\end{array}$ & $\mathrm{LVH}+\mathrm{LAD}+\mathrm{AF}$ & $\begin{array}{l}\text { Increased CT ratio with increased vascularity } \\
\text { with upper lobe diversion }\end{array}$ & $\begin{array}{l}\text { Dilated LV, severe gen. LV } \\
\text { dysfunction. Apical LV clot }\end{array}$ & Alive & Alive \\
\hline 17 & $\begin{array}{l}\text { Rheumatic heart } \\
\text { disease }\end{array}$ & $\begin{array}{l}\text { LA \& LV } \\
\text { enlargement }\end{array}$ & $\begin{array}{l}\text { Increased CT ratio, double contour shadow } \\
\text { on right side }\end{array}$ & $\begin{array}{l}\text { Severe MS and moderate } \\
\text { MR, dilated LA \& LV with } \\
\text { normal function }\end{array}$ & Alive & IUD \\
\hline 18 & $\begin{array}{l}\text { Rheumatic heart } \\
\text { disease }\end{array}$ & $\begin{array}{l}\text { LA enlargement + } \\
\text { RAD }\end{array}$ & $\begin{array}{l}\text { Increased CT ratio with straightening of LHB } \\
+ \text { venous congestion + LA enlargement }\end{array}$ & $\begin{array}{l}\text { Severe MS with pliable } \\
\text { valves }\end{array}$ & Alive & Alive \\
\hline 19 & $\begin{array}{l}\text { Peripartum } \\
\text { cardiomyopathy }\end{array}$ & $\begin{array}{l}\text { LA enlargement } \\
\text { with Poor } \mathrm{R} \text { in } \\
\text { anterior leads }\end{array}$ & $\begin{array}{l}\text { Increased CT ratio with pulmonary } \\
\text { congestion + mild pleural effusion }\end{array}$ & $\begin{array}{l}\text { Dilated LV with severely } \\
\text { depressed function. EF: } 30 \%\end{array}$ & $\begin{array}{l}\text { Maternal } \\
\text { Death }\end{array}$ & IUD \\
\hline 20 & $\begin{array}{l}\text { Peripartum } \\
\text { cardiomyopathy }\end{array}$ & $\begin{array}{l}\text { P-mitrale }+\mathrm{LVH}+ \\
\text { Poor } \mathrm{R} \text { in anterior } \\
\text { leads }\end{array}$ & $\begin{array}{l}\text { Increased CT ratio with pulmonary } \\
\text { congestion }\end{array}$ & EF: $20-25 \%$ & Alive & IUD \\
\hline 21 & $\begin{array}{l}\text { Rheumatic heart } \\
\text { disease }\end{array}$ & P-mitrale + RAD & $\begin{array}{l}\text { Straightening of LHB, double contour shadow } \\
\text { on RHB, upper lobe diversion }\end{array}$ & $\begin{array}{l}\text { Severe MS with calcified MV } \\
\& \text { mild MR }\end{array}$ & Alive & Alive \\
\hline 22 & $\begin{array}{l}\text { Primary } \\
\text { pulmonary } \\
\text { hypertension }\end{array}$ & $\begin{array}{l}\text { RAD + RVH + P- } \\
\text { mitrale }\end{array}$ & $\begin{array}{l}\text { Dilated pulmonary artery, elongated lung } \\
\text { shadow }\end{array}$ & $\begin{array}{l}\text { Moderate } \mathrm{TR}+\text { Moderate } \\
\text { dilated RA and RV }\end{array}$ & Alive & Alive \\
\hline 23 & $\begin{array}{l}\text { Rheumatic heart } \\
\text { disease }\end{array}$ & $\begin{array}{l}\text { Within normal } \\
\text { limits }\end{array}$ & Within normal limits & $\begin{array}{l}\text { Relapsing AML with } \\
\text { thickened MC leaflets (No } \\
\text { MS) }\end{array}$ & Alive & Alive \\
\hline 24 & $\begin{array}{l}\text { Rheumatic heart } \\
\text { disease }\end{array}$ & P-mitrale + RVH & $\begin{array}{l}\text { Increased CT ratio with double density on } \\
\text { RHB }\end{array}$ & $\begin{array}{l}\text { Moderate MS + Moderate } \\
\text { MR, Dilated both LA \& LV }\end{array}$ & Alive & Alive \\
\hline 25 & $\begin{array}{l}\text { Peripartum } \\
\text { Cardiomyopathy }\end{array}$ & $\begin{array}{l}\text { LVH + Poor R in } \\
\text { anterior leads }\end{array}$ & $\begin{array}{l}\text { Increased CT ratio with pulmonary } \\
\text { congestion, obliteration of costophrenic } \\
\text { angles }\end{array}$ & $\begin{array}{l}\text { Dilated LA \& LV with severe } \\
\text { LV dysfunction. }\end{array}$ & Alive & Alive \\
\hline 26 & $\begin{array}{l}\text { Peripartum } \\
\text { cardiomyopathy }\end{array}$ & LVH + LAD & $\begin{array}{l}\text { Increased CT ratio with pulmonary } \\
\text { congestion }\end{array}$ & $\begin{array}{l}\text { Dilated LV with severely } \\
\text { depressed function. EF: } 25 \%\end{array}$ & $\begin{array}{l}\text { Maternal } \\
\text { death }\end{array}$ & Alive \\
\hline 27 & $\begin{array}{l}\text { Rheumatic heart } \\
\text { disease }\end{array}$ & P-mitrale + RVH & $\begin{array}{l}\text { Straightening of LHB, double contour shadow } \\
\text { on RHB, increased venous congestion }\end{array}$ & Dilated both LA \& LV & Alive & Alive \\
\hline 28 & Aortic stenosis & $\begin{array}{l}\text { No specific } \\
\text { changes }\end{array}$ & $\begin{array}{l}\text { Increased CT ratio with straightening of LHB, } \\
\text { prosthetic valve shadow on left sternal border }\end{array}$ & $\begin{array}{l}\text { Prosthetic mitral and aortic } \\
\text { valves with hypertrophy, } \\
\text { normal functioning LV }\end{array}$ & Alive & Alive \\
\hline
\end{tabular}

TABLE 1: Cardiovascular co-morbidity, chest x-ray, ECG, and echo findings, and maternal and fetal outcomes of pregnant women

Abbreviations: AF, Atrial Flutter; CT, Cardiothoracic; EF, Ejection Fraction; LA, Left Atrium; LAD, Left Axis Deviation; LHB, Left Heart Border; LV, Left Ventricle; LVH, Left Ventricular Hypertrophy; MC, Mitral Cusp; MR, Mitral Regurgitation; MS, Mitral Stenosis; RA, Right Atrium; RAD, Right Axis Deviation; RHB, Right Heart Border; RV, Right Ventricle; RVH, Right Ventricular Hypertrophy; TR, Tricuspid Regurgitation 


\section{Discussion}

In our study, the prevalence of cardiac disease in pregnancy was $1.22 \%$. This was consistent with a previous regional study conducted in India [6] and Pakistan [7]. Cardiac disease is the most common non-obstetric maternal death [11].

The most common cardiac disease reported in our study was cardiomyopathy. Twelve out of 28 patients were diagnosed with some form of cardiomyopathy. Peripartum cardiomyopathy is more common generally in a month before or after partum, as opposed to dilated cardiomyopathy, which already presents itself by the second trimester. Mortality in peripartum cardiomyopathy varies from $<2 \%$ to $50 \%$ [11]. Heart failure and arrhythmia are the most common complications associated with cardiomyopathy in pregnancy [11]. There are various risk factors, such as obesity, diabetes, hypertension, and stress, during pregnancy, which is responsible for the increase in cardiomyopathy. Increased maternal age is also a known risk factor [6].

The second most common cardiac disease reported in our study was rheumatic disease. Unlike our study, rheumatic heart disease remains the number one worldwide cause of maternal cardiac complications in pregnancy. Since symptoms of rheumatic fever typically do not present until the fourth or fifth decade, the pathophysiology changes associated with pregnancy may cause as many as $25 \%$ of these women to first experience symptoms during pregnancy [12].

The maternal mortality in our study was $14.6 \%$. This was slightly low as compared to that reported in our neighboring country of India [6] but, still, attention should be paid to this considerably high mortality rate when examined on its own.

While our study adds to the limited data available in Pakistan related to cardiac disease in pregnancy, it has its limitations. First, since this was not a long-term prospective study, fetal outcomes and implications on maternal outcomes were not properly recorded. It also failed to identify risk factors that lead to the development of cardiac diseases.

It is really important to develop a culture of early recognition and close follow-up, which can improve maternal tolerance to increase the cardiovascular burden that is part of the normal physiology in pregnancy. This will not only promote neonatal survival but also fetal growth. There should be separate and individual attention, along with counseling to women who get pregnant with a pre-existing heart disease. By identifying the risk factors and reducing the burden of cardiac disease in pregnancy, we can reduce maternal and fetal mortality.

\section{Conclusions}

In our study, the most common cardiac disease among our patients was peripartum cardiomyopathy followed by rheumatic heart disease in women. However, since it is a small sample size, single-institute study, caution should be taken to apply its result to a broader population. A further large-scale trial is needed to understand cardiac disease in pregnant women in Pakistan. These cardiac diseases are responsible for fetal and maternal adverse outcomes. Every effort at the government, institution, and community levels should be made to create awareness regarding the pre-pregnancy counseling of women with cardiac disease. Every effort should be made to create awareness regarding pre-pregnancy counseling, so that associated fetal and maternal morbidity can be reduced.

\section{Additional Information}

\section{Disclosures}

Human subjects: Consent was obtained by all participants in this study. Ghulam Muhammad Mahar Medical College issued approval GMMMC/18/01/06A. Animal subjects: All authors have confirmed that this study did not involve animal subjects or tissue. Conflicts of interest: In compliance with the ICMJE uniform disclosure form, all authors declare the following: Payment/services info: All authors have declared that no financial support was received from any organization for the submitted work. Financial relationships: All authors have declared that they have no financial relationships at present or within the previous three years with any organizations that might have an interest in the submitted work. Other relationships: All authors have declared that there are no other relationships or activities that could appear to have influenced the submitted work.

\section{References}

1. Weiss BM, von Segesser LK. Alon E, Seifert B, Turina MI: Outcome of cardiovascular surgery and pregnancy: a systematic review of the period 1984-1996. Am J Obstet Gynecol. 1998, 179:1643-1653. 10.1016/S00029378(98)70039-0

2. Bush N, Nelson-Piercy C, Spark P, Kurinczuk JJ, Brocklehurst P, Knight M (on behalf of UKOSS): Myocardial infarction in pregnancy and postpartum in the UK. Eur J Prev Cardiol. 2013, 20:12-20. 


\section{Cureus}

$10.1177 / 1741826711432117$

3. Nanda S, Nelson-Piercy C, Mackillop L: Cardiac disease in pregnancy. Clin Med (Lond). 2012, 12:553-560.

4. Thompson JL, Kuklina EV, Bateman BT, Callaghan WM, James AH, Grotegut CA: Medical and obstetric outcomes among pregnant women with congenital heart disease. Obstet Gynecol. 2015, 126:346-354. 10.1097/AOG.0000000000000973

5. Franklin WJ, Benton MK, Parekh DR: Cardiac disease in pregnancy. Tex Heart Inst J. 2011, 38:151-153.

6. Konar H, Chaudhuri S: Pregnancy complicated by maternal heart disease: a review of 281 women . J Obstet Gynaecol India. 2012, 62:301-306. 10.1007/s13224-012-0220-2

7. Wasim T, Amer W, Majrooh A, Siddiq S: Foetomaternal outcome of pregnancy with cardiac disease . J Pak Med Assoc. 2008, 58:175-178.

8. Azibani F, Sliwa K: Peripartum cardiomyopathy: an update. Curr Heart Fail Rep. 2018, 15:297-306. 10.1007/s11897-018-0404-X

9. Isogai T, Kamiya CA: Worldwide incidence of peripartum cardiomyopathy and overall maternal mortality . Int Heart J. 2019, 60:503-511. 10.1536/ihj.18-729

10. Munir R, Hussain S, Kayani AM: Peripartum cardiomyopathy in a Pakistani cohort. J Coll Physicians Surg Pak. 2016, 26:740-743.

11. Schaufelberger M: Cardiomyopathy and pregnancy. Heart. 2019, 105:1543-1551. 10.1136/heartjnl-2018313476

12. Mitchelson JB, Cleveland DE: Optimal management of a pregnant patient with rheumatic heart disease . Kans J Med. 2018, 11:80-82. 